\title{
Muon colliders and neutrino factories
}

\author{
Daniel M. Kaplan ${ }^{1, a}$, on behalf of the MAP and MICE Collaborations \\ ${ }^{1}$ Illinois Institute of Technology, Chicago, Illinois 60616, USA
}

\begin{abstract}
Muon colliders and neutrino factories are attractive options for future facilities aimed at achieving the highest lepton-antilepton collision energies and precision measurements of Higgs boson and neutrino mixing matrix parameters. The facility performance and cost depend on how well a beam of muons can be cooled. Recent progress in muon cooling design studies and prototype tests nourishes the hope that such facilities could be built starting in the coming decade. The status of the key technologies and their various demonstration experiments is summarized. Prospects "post-P5" are also discussed.
\end{abstract}

\section{Introduction: Why Muon Colliders are Obviously Best - and Why Not}

Muon colliders offer the only way to study matter with well-understood leptonic probes both at comparable and at smaller distances than those accessible to the LHC. However, despite the obvious muon advantages at high energies, linear or circular electron-positron colliders are currently under serious consideration to follow up the discovery of the Higgs boson, while muon colliders are not. Muon advantages stem mainly from its 200 -times greater mass:

(1) Radiative processes (inversely proportional to the fourth power of lepton mass) are greatly suppressed, enabling the use of storage rings and compact recirculating accelerators (Fig. 1).

(2) So are the "beamstrahlung" interactions that limit $e^{+} e^{-}$-collider luminosity as energy increases [1].

(3) The smaller size of a muon collider (Fig. 2) eases the siting issues and suggests that the cost may be less as well.

(4) The cross-section ratio for $s$-channel lepton-antilepton annihilation to scalar bosons, $\sigma_{\mu} / \sigma_{e}=$ $\left(m_{\mu} / m_{e}\right)^{2}=4.3 \times 10^{4}$, gives the muon collider unique access to precision Higgs measurements [2-5]. For example, at the $\approx 125 \mathrm{GeV} / c^{2}$ mass measured by ATLAS and CMS [6], only a muon collider can directly observe the (4 MeV) width and lineshape of a Standard Model Higgs boson [2] (Fig. 3 left).

(5) Furthermore, should the Higgs have closely spaced partner states at higher mass, only a muon collider has sufficient mass resolution to distinguish them (Fig. 3 right). (This is a possible feature of supersymmetry as well as other new-physics scenarios.)

\footnotetext{
ae-mail: kaplan@iit.edu
} 


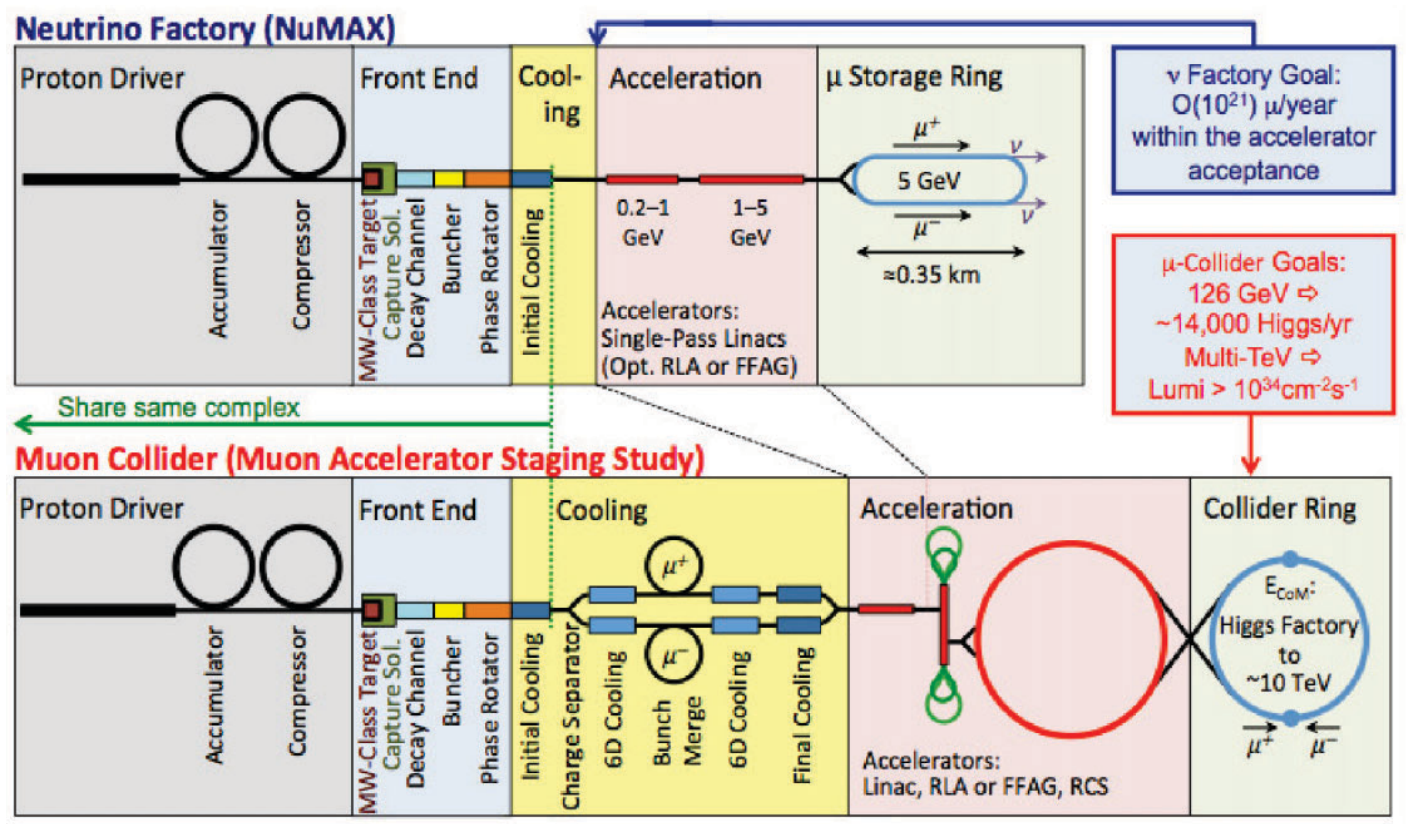

Figure 1. Neutrino factory and muon collider conceptual block diagrams. The two types of facilities share a number of elements in common (high-power, medium-energy "Proton Driver" and MW-capable target, muon cooling, muon acceleration and storage rings) and, up through the "Initial Cooling," are nearly identical.

(6) For the highest lepton energies the muon collider has by far the least operating cost of any proposed approach, and (because of the lack of beamstrahlung) the highest luminosity within $1 \%$ of the energy peak (Fig. 4).

Of course, linear $e^{+} e^{-}$colliders have received enormous attention and resources in recent decades, bringing them to a state of substantial technological readiness. And, due to their $2.2 \mu$ s lifetime, muons must be cooled using novel technology before they can be accelerated to collider energy. This lifetime disadvantage has delayed the general acceptance of muon colliders, inhibiting the R\&D process required to demonstrate that the disadvantage can be overcome.

\section{Neutrino Factories}

On the other hand, their $O(\mu \mathrm{s})$ lifetime and simple, well-understood, purely leptonic decay dynamics make muons ideal sources for neutrino beams of unprecedented purity and precision. This realization led to the neutrino factory idea [7], which has now been brought to the brink of feasibility by the IDSNF project [8]. On the list of "go/no-go" feasibility demonstrations, only the Muon Ionization Cooling Experiment [9] (MICE) remains to be completed; its progress and prospects are discussed below. Figure 5 shows that the neutrino factory has the best precision of any proposed facility for measuring the $C P$-asymmetry parameter $\delta$ of the PMNS neutrino mixing matrix, with sensitivity rivaling that in the quark sector - a reasonable sensitivity goal in order to probe the GUT-scale physics that may link the quark and lepton sectors. Its capability for precision measurement of the PMNS matrix gives the 


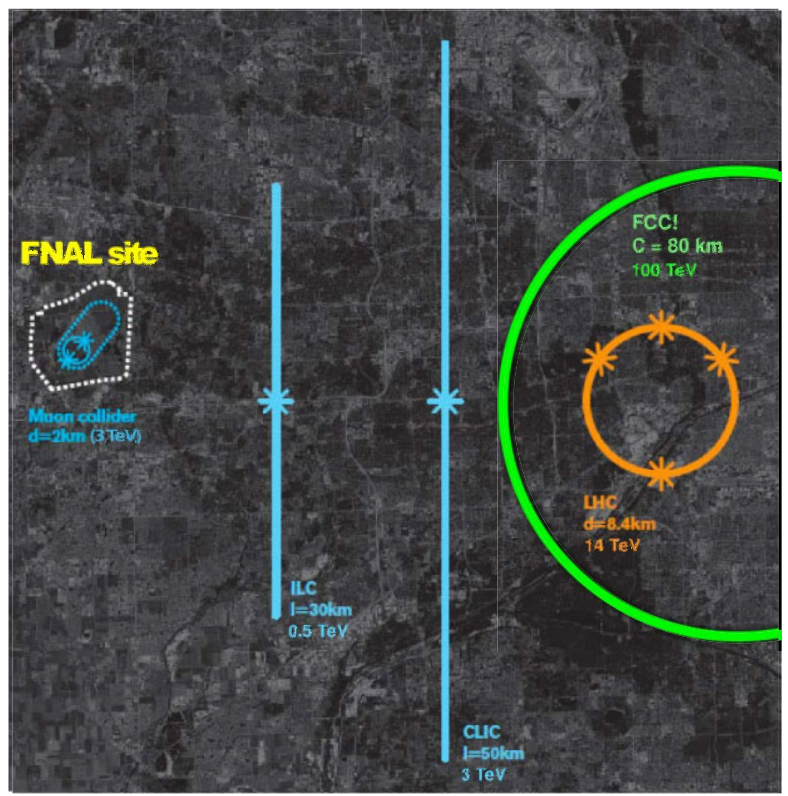

Figure 2. Sizes of various proposed colliders compared with FNAL site. Unlike the others, a muon collider with $\sqrt{s}>3 \mathrm{TeV} f$ ts on existing sites.
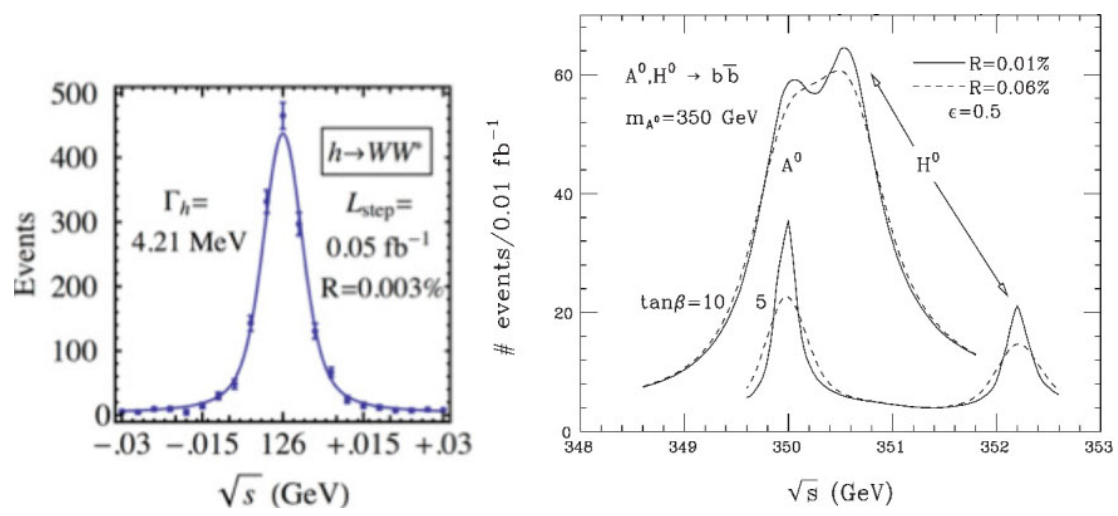

Figure 3. (left) Example of Higgs resonance scan using s-channel production in a muon collider Higgs Factory; (right) resolving scalar and pseudoscalar supersymmetric Higgs partners at a higher-energy muon collider for two possible values of the supersymmetric parameter $\tan \beta[4]$. ( $\mathrm{R}$ is the the collision energy spread.)

neutrino factory the best reach for $\mathrm{f}$ nding possible new physics beyond three-f avor mixing. It is thus the logical follow-on facility to LBNF.

\section{MASS Facility Staging Plan}

The Muon Accelerator Staging Study [10] (part of the US national Muon Accelerator Program, MAP [11]) has outlined a scenario of neutrino factory and muon collider construction, presumed 

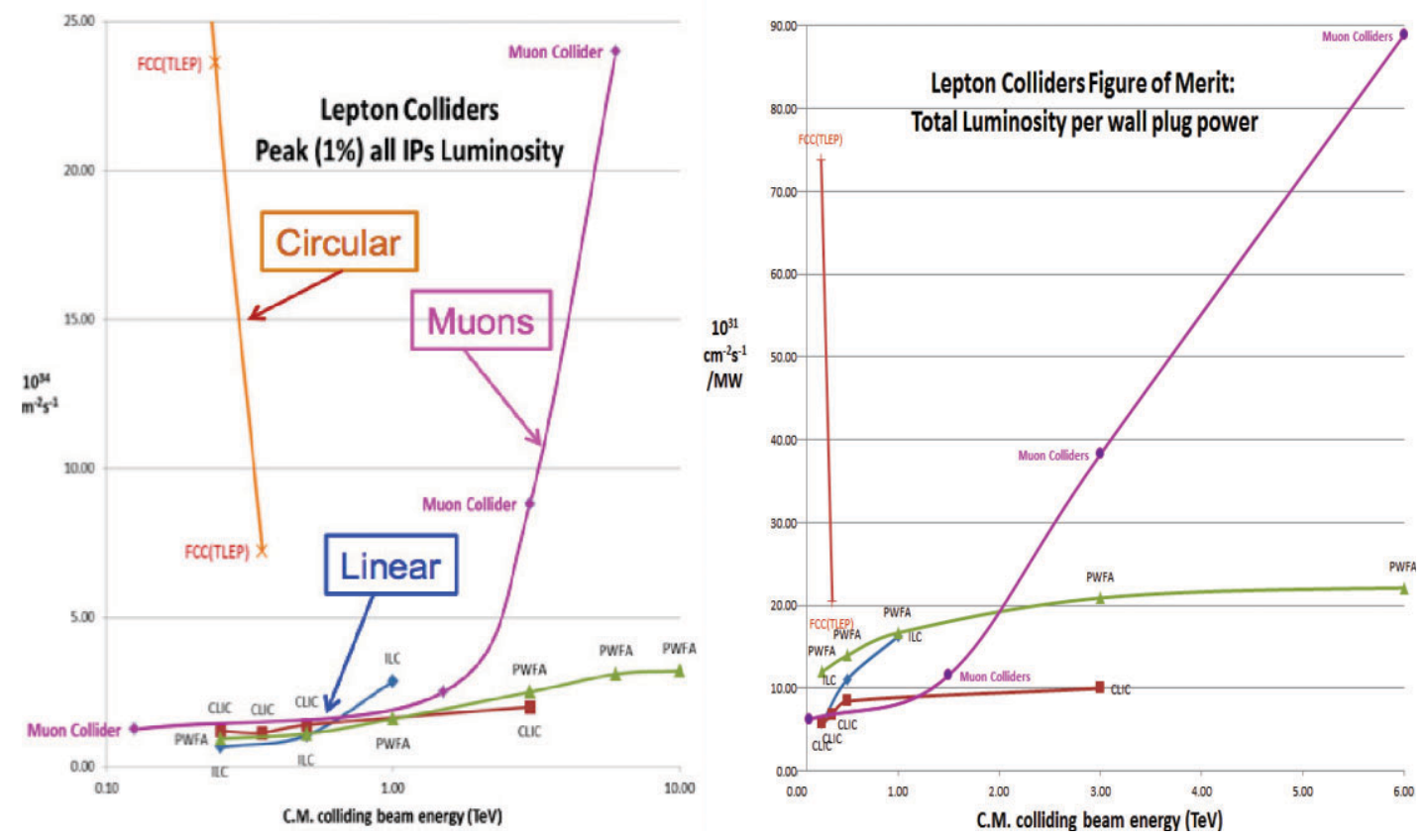

Figure 4. (left) Luminosity within $1 \%$ of peak $\ell^{+} \ell^{-}$energy, and (right) luminosity per unit wall-plug power, for various proposed collider technologies and lepton choices.

to be at Fermilab, with successive upgrades, wherein each step is reasonably affordable and brings improved physics reach.

(1) The plan starts with the proposed nuSTORM [12] pion-injected muon storage ring short-baseline experiment, aimed at a definitive test of the sterile-neutrino interpretation of the results from LSND, MiniBooNE, reactor experiments, etc., as well as precision neutrino cross-section measurements in the energy range crucial to LBNF. NuSTORM requires no new technology and no R\&D, so could be built immediately. It will afford the opportunity to develop instrumentation for, and experience with, muon storage ring neutrino sources which will be applicable to successor neutrino factories.

(2) The next step, NuMAX, is an initial long-baseline neutrino factory at Fermilab optimized for a detector at the Sanford Underground Research Facility (SURF) in South Dakota, with physics reach exceeding that of LBNF (Fig. 5). NuMAX is conceived to start without muon cooling and with a sub-megawatt beam and target.

(3) The follow-on, NuMAX+, facility adds a limited amount of muon cooling and higher-power beam and target, for more than an order-of-magnitude increase in neutrino intensity.

Beyond these neutrino-oriented facilities, a series of muon colliders could be built, including:

(4) A Higgs Factory muon collider delivering $>10^{4}$ Higgs events per year with exquisite energy resolution.

(5) A multi-TeV muon collider $(\sqrt{s} \lesssim 10 \mathrm{TeV})$ offering the best performance and least cost and power consumption of any lepton collider in this energy range (Fig. 4). 
ICNFP 2014
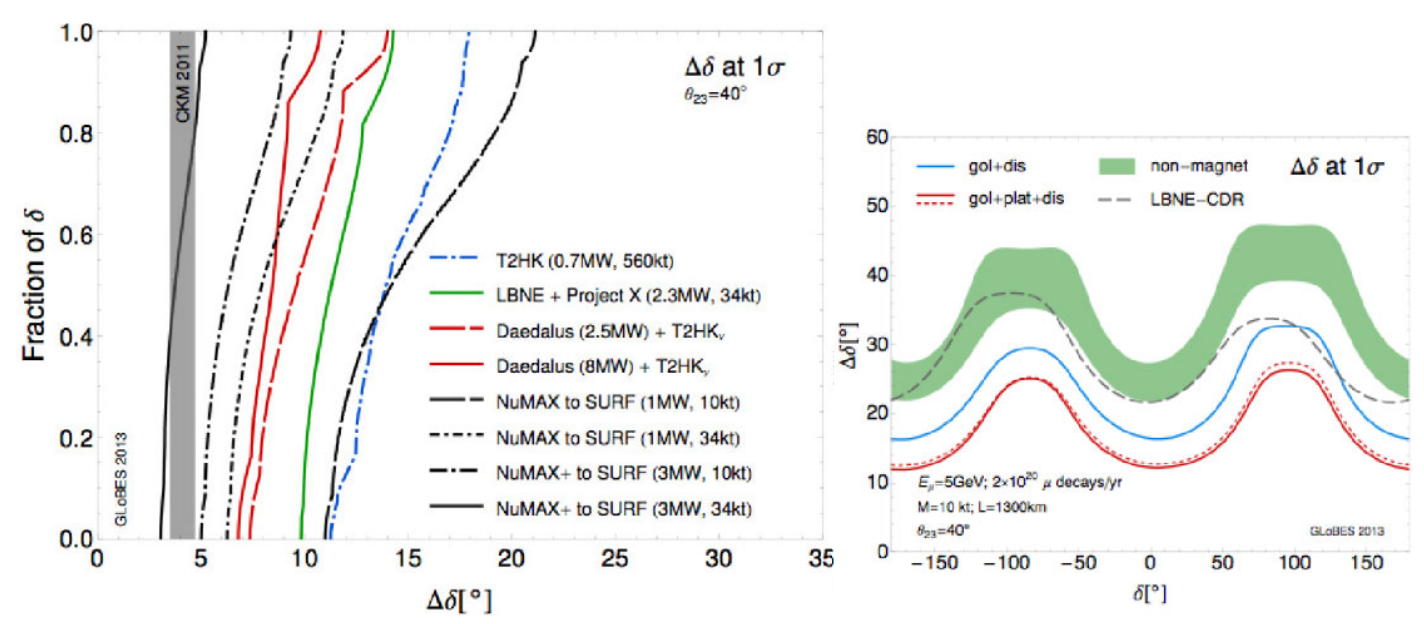

Figure 5. (left) Comparison of $C P$-violation reach of proposed future neutrino facilities, in terms of the fraction of the range of the $C P$-violating phase $\delta$ of the PMNS neutrino mixing matrix over which it can be measured to a given precision, and (right) comparison of precision achievable in measuring $\delta$ vs. its true value. Of all proposed future neutrino facilities, the neutrino factory is seen to have the best $C P$ reach as well as the best precision on $\delta$.

Of course, depending on future physics discoveries and world-wide HEP funding exigencies, the elements of this scheme may not all be built, or not all at Fermilab, and not necessarily in this order. This is also recognized as a rather ambitious plan, extending as it does some 20 to 30 years or more into the future. We consider below how it could be viewed in light of the P5 recommendations [13].

The following sections provide a brief overview of these muon facilities as well as some flavor of the R\&D that has been pursued in order to bring them closer to fruition. A more extensive and detailed review may be found in [14].

\section{Technical Challenges}

Muon storage-ring facilities present four main technical challenges requiring novel solutions: (1) producing enough muons; (2) cooling the muon beams to enable high intensity and luminosity; (3) rapidly accelerating the beams; and (4) storage-ring designs that can deliver small enough $\beta^{*}$ at the collision points - or alternatively, the needed neutrino-beam pointing and timing characteristics - while coping with the high rate of decay electrons. Solutions have been devised for all four of these challenges. While space constraints prevent a detailed discussion, we here briefly comment on each.

\section{Muon production}

An issue potentially limiting the collider luminosity or neutrino flux that can be achieved is the production of muons in sufficient quantity. The only method that appears suitable is production and decay of low-energy $(<1 \mathrm{GeV})$ pions via fixed-target collisions of a megawatt-scale proton beam. Carbon targets have been discussed for beam power up to about $1 \mathrm{MW}$ [15]. For the one-to-several MW range, a free mercury-jet target has been shown feasible by the MERIT experiment, conducted in 2007 at CERN [16]. Much subsequent work has gone into optimizing the configuration of the target region and the solenoids (with up to $3 \mathrm{GJ}$ stored energy) that serve to capture the produced pions and their decay muons $[17,18]$. Recent concept drawings are shown in Fig. 6. 

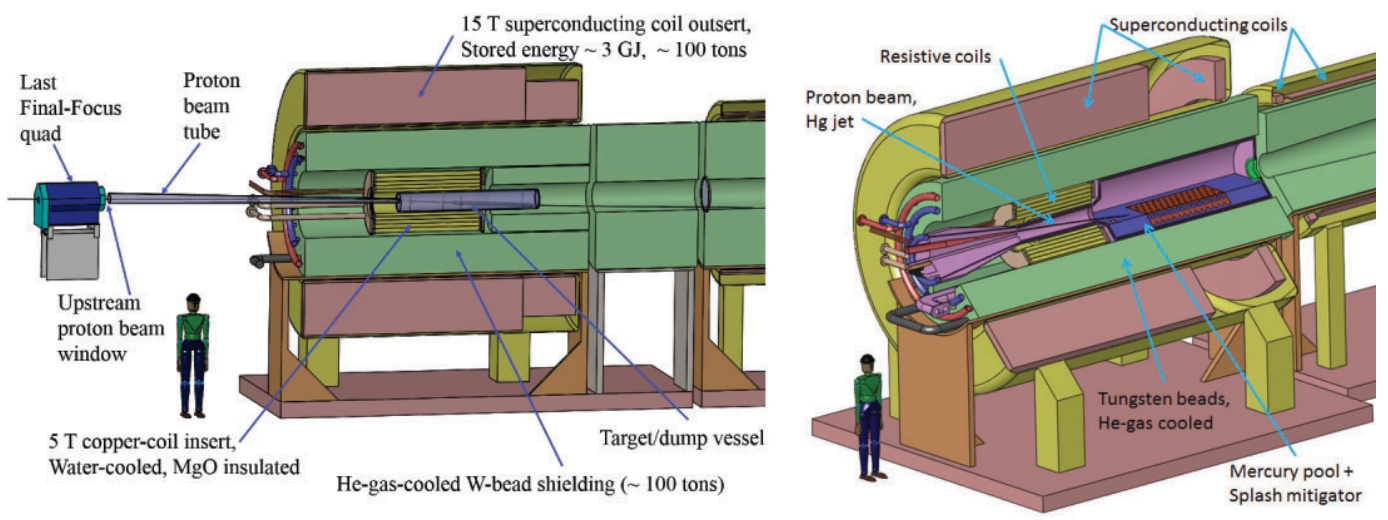

Figure 6. Sketch of Target System concept: (left) $\_1 \mathrm{MW}$ version, with C target [15]; (right) $4 \mathrm{MW}$ version, with Hg-jet target and Hg-pool beam dump [18].

\section{Muon cooling}

Muon cooling is introduced in some detail in the next section. It is required in order to achieve the luminosity goals for a muon collider, and it is cost-effective for a neutrino factory in that it allows the apertures of the acceleration systems to be reduced. Although earlier neutrino factory work assumed only transverse cooling, recent work has emphasized the utility of muon cooling in all six phasespace dimensions in order to optimize the facility design as a whole by allowing the proton linac to be efficiently reused for the muon beam [19].

It is important to distinguish the six-dimensional cooling factor of several million required in order to achieve high collider luminosity from the much more modest $\sim 10$ to 50 cooling factor that suffices for a high-intensity neutrino factory. Indeed, a neutrino factory built initially with no muon cooling whatsoever is competitive with proposed future facilities based on neutrinos from pion decay (see Fig. 5). This is one reason why a staged approach such as that discussed above is sensible.

\section{Rapid muon acceleration}

To ensure adequate muon survival $(\stackrel{\sim}{\sim} 10 \%$ decay losses), acceleration must occur at high average gradient. At the low energy ( $120 \mathrm{MeV}$ kinetic) that is optimal for ionization cooling, only a linac has sufficient performance. Once the muons have been accelerated to a few $\mathrm{GeV}$, there is sufficient time dilation for recirculating accelerators (with substantially lower costs per $\mathrm{GeV}$ ) to be used: RLAs, FFAGs, and rapid-cycling synchrotrons, as schematically indicated in Fig. 1. These technologies are challenging to implement for muon applications since, even after cooling, emittances are larger than those in electron and proton machines. At lower energies solenoid focusing is therefore preferred. At higher energy, "dogbone" RLAs (with quadrupole focusing) ease switchyard design compared to the more conventional racetracks [20]. The number of passes through each RLA can be increased by means of pulsed quadrupoles [21], and further cost-efficiency can be achieved via two-pass arcs [22].

At the highest energies rapid-cycling synchrotrons become favorable. These might employ novel fast-ramping ( $\sim \mathrm{kHz})$ dipoles, with thin, grain-oriented steel laminations [23] or "hybrid" laminations composed of Si steel with FeCo pole tips [24]. A design has been studied in which such pulsed mag- 
nets alternate with fixed-field $8 \mathrm{~T}$ superconducting dipoles, accelerating muons from 30 to $750 \mathrm{GeV}$ in two rings each the size of the Tevatron ${ }^{1}[25,26]$.

\section{Storage-ring design}

A neutrino factory requires an oblong ("racetrack") storage ring, with long straight sections that direct decay neutrinos towards near and far detectors. A series of designs have been developed at various energies, starting in the earliest feasibility studies [27, 28]. Most recently, the IDS-NF study [8] worked out a design for a $10 \mathrm{GeV}$ decay ring, which can easily be scaled to the $5 \mathrm{GeV}$ that is optimal for the Fermilab-SURF baseline.

On the other hand, a muon collider storage ring should have minimal straight sections and be as small as possible, in order to maximize the number of turns made by the muons, and hence the number of collisions before they decay. This calls for high bending field; $10 \mathrm{~T}$ is typical. Designs have been worked through for a $125 \mathrm{GeV}$ Higgs Factory [29] with $4 \mathrm{MeV}$ energy spread $(\delta E / E \approx 0.003 \%)$ at the IP and for 1.5 [30] and 3.0 TeV [31] collision energies. These employ magnets enclosing tungsten beam-pipe liners in order to absorb decay electrons. At the highest energies care must be taken in order to limit the radiation exposure of people living near locations where the "neutrino pancake" due to muon decays in the ring intersects the earth's surface. Thus in the $3 \mathrm{TeV}$ design, combined-function magnets are used in the arcs instead of quadrupoles in order to have bending field everywhere.

\section{Muon Cooling}

A key ingredient in most of the muon facilities discussed here is muon cooling - an area in which there has been important recent progress. Established (electron, stochastic, and laser) beam-cooling methods take minutes to hours and so are ineffective on the microsecond timescale of the muon lifetime. However, the muon's penetrating character enables rapid cooling via ionization energy loss [32, 33]. At sufficiently high energy (e.g., a Higgs Factory or higher-energy muon collider), optical stochastic cooling $[34,35]$ can also be considered and may enable higher luminosity or reduced energy spread. (So-called "frictional" cooling has also been considered [36] but appears to be inapplicable to high-intensity stored muon beams and high-luminosity colliders.)

An ionization-cooling channel comprises energy absorbers and radio-frequency (RF) accelerating cavities placed within a suitable focusing magnetic lattice. In the absorbers the muons lose both transverse and longitudinal momentum, and the RF cavities restore the lost longitudinal momentum. In this way, the large initial divergence of the muon beam can be reduced. Within an energy-absorbing medium, normalized transverse emittance depends on path length $s$ as [33]

$$
\frac{d \epsilon_{n}}{d s} \approx-\frac{1}{\beta^{2}}\left\langle\frac{d E_{\mu}}{d s}\right\rangle \frac{\epsilon_{n}}{E_{\mu}}+\frac{1}{\beta^{3}} \frac{\beta_{\perp}(0.014)^{2}}{2 E_{\mu} m_{\mu} L_{R}},
$$

where $\beta c$ is the muon velocity, $E_{\mu}$ the muon energy in $\mathrm{GeV}, m_{\mu}$ its mass in $\mathrm{GeV} / c^{2}, \beta_{\perp}$ the lattice betatron function, and $L_{R}$ the radiation length of the medium. A portion of this cooling effect can be transferred to the longitudinal phase plane ("emittance exchange") by placing suitably shaped absorbers in dispersive regions of the lattice [33], by using momentum-dependent path-length within flat absorbers, or within a homogeneous absorber that fills the lattice [37]. (Longitudinal ionization cooling per se, which would entail operation at momenta above the minimum of the ionization curve, so as to have negative feedback in energy, is impractical due to energy-loss straggling [33]).

\footnotetext{
${ }^{1}$ whose circumference was $6.3 \mathrm{~km}$.
} 


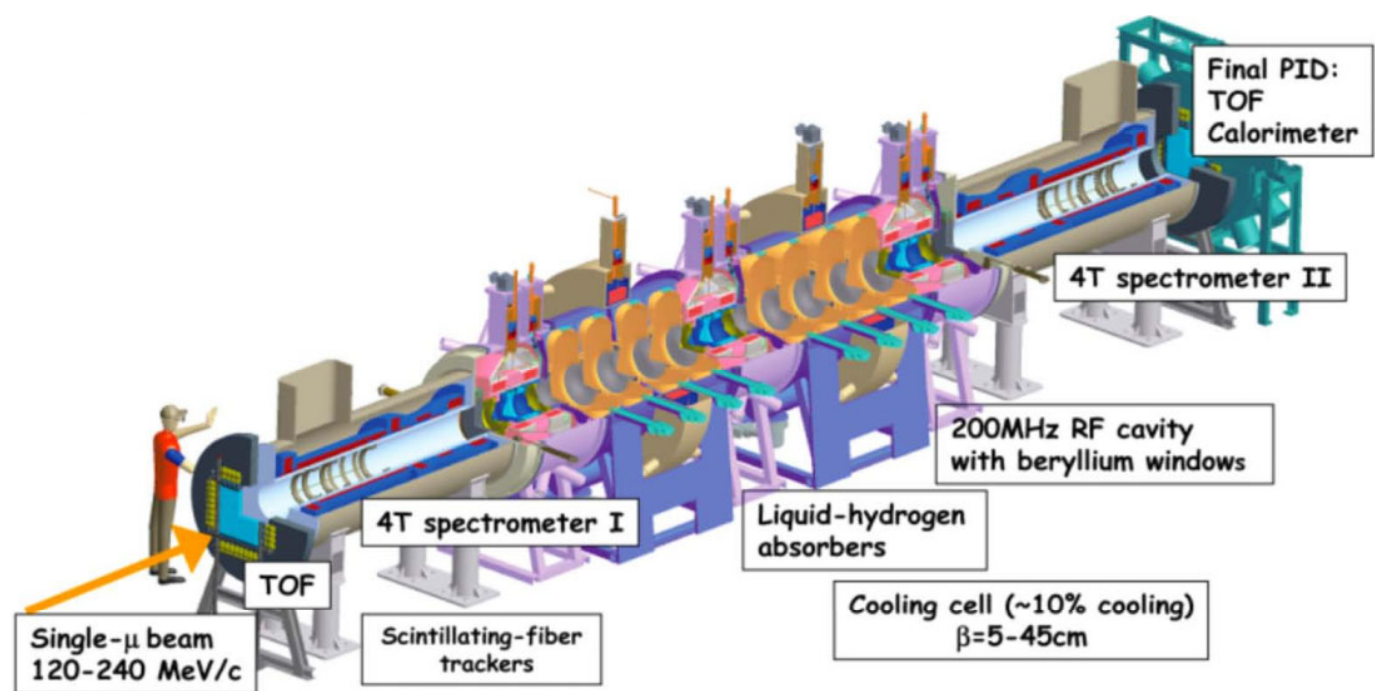

Figure 7. Three-dimensional cutaway rendering of MICE apparatus (see text) as originally envisioned: individual muons entering at lower left are measured by time-of-flight (TOF) and Cherenkov counters and a solenoidal tracking spectrometer; then, in cooling section, alternately slowed in $\mathrm{LH}_{2}$ absorbers and reaccelerated by RF cavities, while focused by a lattice of superconducting solenoids; then remeasured by a second solenoidal tracking spectrometer, and their muon identity confirmed by TOF detectors and calorimeters. The cooling section includes three pairs of small "focus coil" magnets surrounding the absorbers and two large "coupling coil" magnets surrounding the RF cavities, comprising one complete lattice cell of the Feasibility Study-II initial cooling lattice, plus one additional absorber and focus-coil pair for symmetry.

The two terms of Eq. 1 represent, respectively, muon cooling by energy loss and heating by multiple Coulomb scattering. Setting them equal approximates the equilibrium value of the emittance, $\epsilon_{n, e q}$, at which the cooling rate reaches zero, and beyond which a given lattice cannot cool. Since the heating term scales with $\beta_{\perp} / L_{R}$, to achieve a low $\epsilon_{n, e q}$ requires low $\beta_{\perp}$ at the absorbers. Superconducting solenoids, which can give $\beta_{\perp} \ll 1 \mathrm{~m}$, are thus the focusing element of choice. Likewise, low-Z absorber media are favored, the best being hydrogen (approximately twice as effective for cooling as the next best materials, helium and $\mathrm{LiH} \mathrm{[38]).}$

It is the absorbers that cool the beam, but for typical "real-estate" accelerating gradients $\left(\approx 10 \mathrm{MeV} / \mathrm{m}\right.$, to be compared with $\left\langle d E_{\mu} / d s\right\rangle \approx 30 \mathrm{MeV} / \mathrm{m}$ for liquid hydrogen [39]), it is the RF cavities that determine the length of the cooling channel (see e.g. Fig. 7). The achievable RF gradient thus determines how much cooling is practical before an appreciable fraction of the muons have decayed. High-gradient vacuum RF cavities (normal-conducting due to the magnetic field in which they must operate) for muon cooling are under development, as is an alternative approach: cavities pressurized with hydrogen gas, thus combining energy absorption and reacceleration [40]. In the first cooling stages the large size of the uncooled beam requires relatively low RF frequency. As the beam is cooled, focal lengths must be shortened in order to reduce the equilibrium emittance, and cavity frequencies and gradients can be increased. Goals are $\gtrsim 15 \mathrm{MV} / \mathrm{m}$ at $201 \mathrm{MHz}$ in $\approx 2 \mathrm{~T}$ fields, and $\approx 25 \mathrm{MV} / \mathrm{m}$ at $805 \mathrm{MHz}$ in $\approx 3 \mathrm{~T}$. Despite early evidence that breakdown limits cavity performance in high magnetic fields, promising results on meeting these goals are now coming from work at the Fermilab MuCool Test Area (MTA) [41]. 


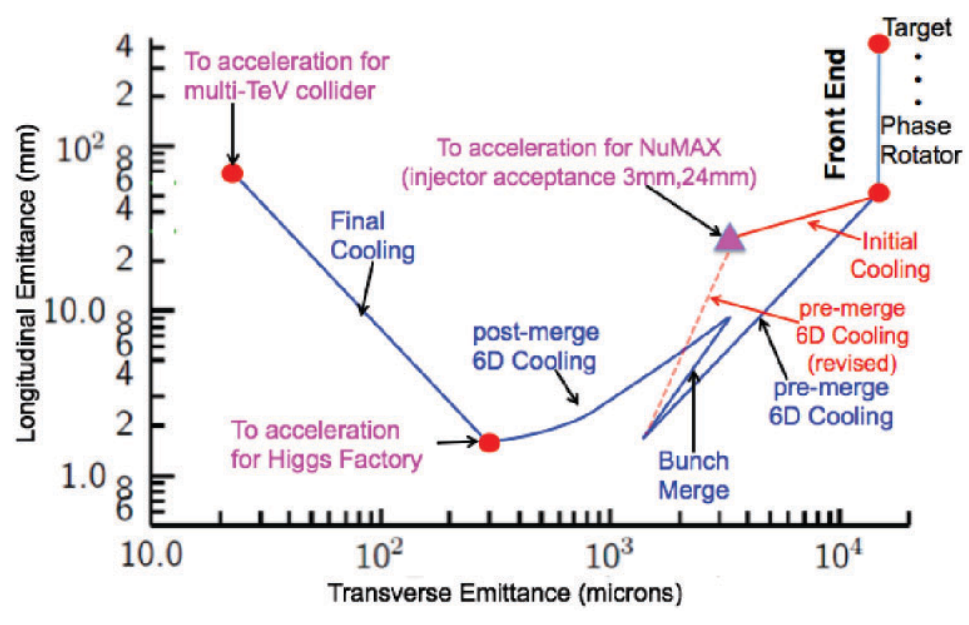

Figure 8. Plot of emittance evolution path in longitudinal and transverse planes for representative muon collider cooling scenarios, showing, in blue, the path without a "dual-use" linac and, in orange, that with such a linac. In the dual-use linac scenario [19], the relativistic part of the Proton Driver $\mathrm{H}^{-}$linac is reused for medium-energy muon acceleration.

In the cooling term of Eq. 1, the fractional decrease in normalized emittance is proportional to the fractional energy loss, thus (at $200 \mathrm{MeV} / \mathrm{c}$ ) cooling in one transverse dimension by a factor $1 / e$ requires $\sim 50 \%$ energy loss and replacement. Ionization cooling thus favors low beam momentum, despite the relativistic increase of muon lifetime with energy, due to the increase of $d E / d s$ for momenta below the ionization minimum [39], the greater ease of beam focusing, and the lower accelerating voltage required. Most muon-cooling designs have therefore used momenta in the range $150-400 \mathrm{MeV} / c$. This is also the momentum range in which the pion-production cross section from thick targets tends to peak and is thus optimal for muon production as well as cooling. The cooling channel of Fig. 7, for example, is optimized for a mean muon momentum of $200 \mathrm{MeV} / c$.

\section{Muon collider cooling scenarios}

Figure 8 shows the emittance evolution in a typical muon collider cooling scenario. The muon beam emerging from decays of pions produced at the target is captured in solenoids, and bunched and "phase-rotated" in order to reduce its energy spread at the expense of increased length [42]. The bunches then proceed to the initial 6D cooling channel, a candidate for which is the so-called "FOFO Snake" [43] (Fig. 9 top), which is designed to cool both positive and negative muons simultaneously but has limited capability to reach low $\beta_{\perp}$. Following Initial Cooling the $\mu^{+}$and $\mu^{-}$bunches will need to be separated for further $6 \mathrm{D}$ cooling, then recombined and coalesced before acceleration and storage; candidate designs to carry out these operations exist [44].

In Fig. 8 , the red point at $\approx 1.5 \mathrm{~mm}$ longitudinal emittance is the cooling output point for a Higgs Factory, which needs exquisite energy resolution and, hence, the minimum achievable longitudinal emittance. This is estimated to be limited (in the "VCC" design, at least) to $\approx 1.5 \mathrm{~mm}$ due to spacecharge effects [45]. Two cooling approaches (HCC and VCC) have been shown effective in simulation studies aimed at reaching that output point. The VCC ("Vacuum Cooling Channel") evolved from the "Guggenheim" scheme employing helical channels with bending radii large compared to their 

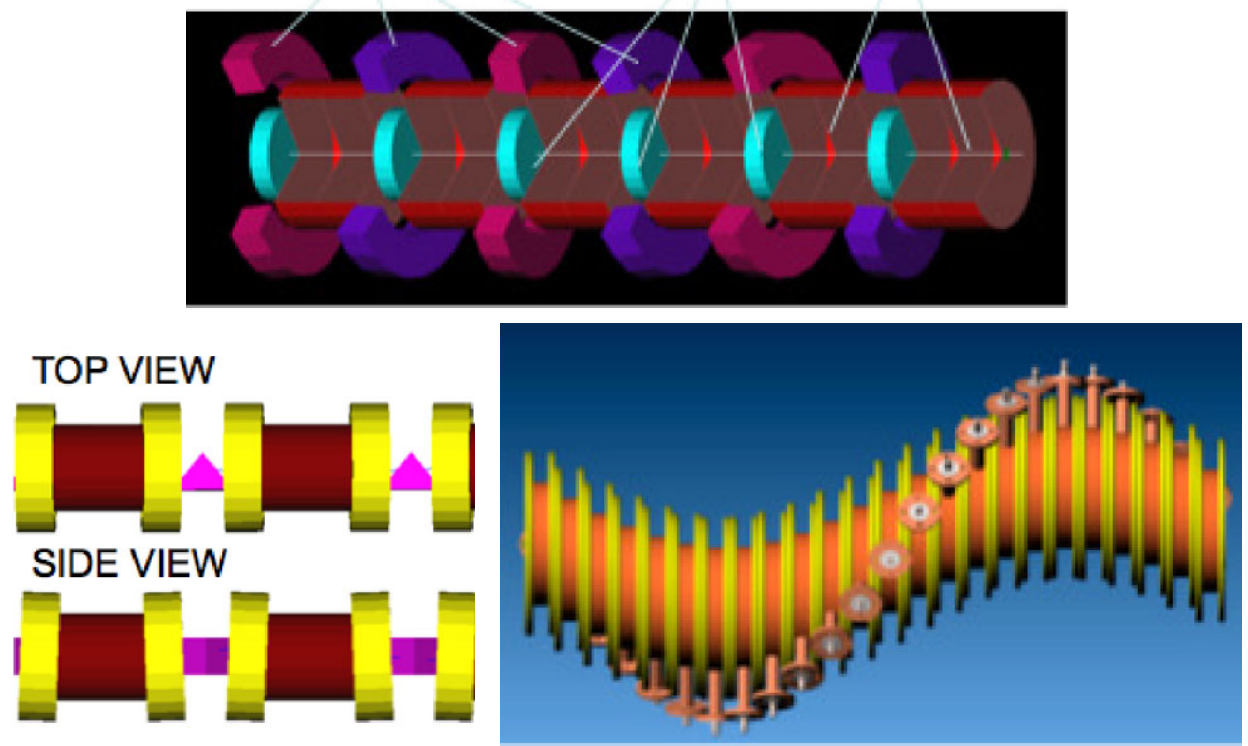

Figure 9. Schematic diagrams of (top) FOFO Snake cooling channel section; (bottom-left) VCC cooling channel section, with superconducting coils in yellow, RF cavities in brown, and wedge absorbers in magenta; (bottomright) schematic of HCC cooling channel section, with coils in yellow, cavities in orange, and RF feeds in salmon.

channel bore, the dipole field components being supplied by tilted thin-lens superconducting solenoid coils [46]. (The Guggenheim evolved from cooling rings [47], which were shown to work but had injection-kicker issues.) The realization that engineering such a structure would be challenging led to the current VCC scheme (Fig. 9 left), which has very similar simulated performance but in a more straightforward beamline geometry [48].

The competing HCC ("Helical Cooling Channel") design [37] (Fig. 9 right) is a helical structure with bore diameter comparable to the bend radius, and is designed to operate with high-pressure gaseous hydrogen distributed throughout. The HCC is believed to work at lower longitudinal emittance than the VCC [49], which might enable a Higgs Factory with even lower energy spread. Additional innovative features of the HCC include RF cavities incorporating dielectric-ring loading for size reduction and helical solenoid magnets composed of current rings that follow the helical paths of the muons [50]. While pressurized cavities suppress breakdown [51], loading of the cavity by ionization electrons was anticipated to be problematic in pure hydrogen. A dedicated R\&D program at the MTA showed that doping the hydrogen with a percent-level admixture of dry air suffices to suppress this plasma loading, allowing operation at muon collider intensities [52].

\section{Final Cooling}

For a multi-TeV muon collider, the longitudinal emittance at the Higgs Factory cooling output point is much smaller than necessary, while the transverse emittance is too large for the desired $O\left(10^{34}\right)$ luminosity. This emittance mismatch is alleviated via "Final Cooling," in which the muon energy is 


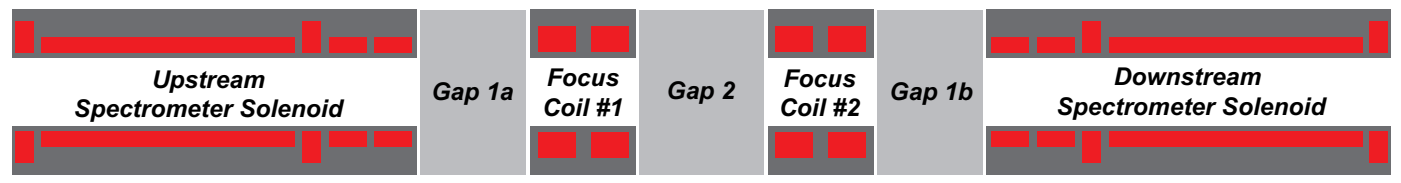

Figure 10. Generic schematic for MICE Final Cooling Demonstration, containing three gaps into which RF cavities and/or absorbers may be placed.

allowed to fall in order to take advantage of the rising $d E / d x$ curve at low energy, and the coolingchannel equilibrium emittance is further reduced by means of small-bore 30-40 T solenoids enclosing $\mathrm{LH}_{2}$ absorbers [53]. Such magnets appear to be feasible and are being developed by NHMFL [54] among others [55]. Alternatives to Final Cooling have also been discussed, incorporating, e.g., "Parametric Ionization Cooling" [56] or a "potato slicer" emittance exchanger [57].

\section{MICE}

The Muon Ionization Cooling Experiment, after delays associated with building large superconducting magnets to be cooled by closed-cycle cryocoolers, is on track to take first measurements with absorbers in the beam in 2015. One "lesson learned" (which was already obvious to the experts some years ago) is to use large helium refrigerators in any real cooling channel - although this option was unavailable to us at Rutherford Appleton Laboratory (RAL), where MICE is sited. A second is to move to higher RF frequency so as to reduce the transverse size of components. Simulation studies have now shown that $325 \mathrm{MHz}$ RF cavities (rather than the $201 \mathrm{MHz}$ ones used in MICE) have sufficient aperture, even at the large $O(10 \pi) \mathrm{mm} \cdot \operatorname{rad} \mathrm{RMS}$ normalized transverse emittance of an early-stage muon cooling lattice. A third lesson is to avoid whenever possible large ("coupling coil") superconducting magnets surrounding the cavities (see Fig. 7), and cooling lattices without such coils have now been developed and shown to deliver good performance [48].

The principle of MICE has been to develop very precise emittance measurement techniques, with a low enough beam intensity that each muon can be tracked individually, so as to avoid the need for a long and expensive cooling section. Thus MICE as originally proposed [9] (Fig. 7) included just one lattice cell of the $201 \mathrm{MHz}$ lattice from Neutrino Factory Feasibility Study-II [28]. As of this (Oct., 2014) writing, with the US "P5" committee having recommended an early termination of MICE [13], a new and simpler lattice is being devised in order to obviate the need for the coupling coils. A generic diagram of the new arrangement, shown in Fig. 10, is more reminiscent of recent lattice designs, such as that of the IDS-NF [8], than of the Study II design. Preliminary simulation studies indicate a transverse cooling factor on the order of several percent, easily measurable in MICE given the $0.1 \%$ emittance resolution provided by the scintillating-fiber tracking systems [58] of the input and output solenoidal spectrometers. The MICE ionization cooling demonstration using the arrangement of Fig. 10 is now scheduled for data-taking by 2017.

\section{Conclusions and Perspective}

The muon collider/neutrino factory intellectual journey has been an exciting and a fascinating one, starting from the earliest suggestions [59], accruing important innovations [60], and culminating in the sophisticated simulation studies of today [48]. Indeed, we have reached a point at which the 
muon collider and neutrino factory concepts, a priori seemingly unlikely, now increasingly appear feasible. Moreover, the neutrino factory has been shown to be the most powerful way to study the only non-Standard Model physics that is definitively established, neutrino oscillation.

Should LHC discover a new scale of phenomena above $1 \mathrm{TeV}$, a muon collider will be the obvious way to study it with precision. ${ }^{2}$ Absent such discovery, construction of a large-scale stored-muon facility may be farther off in the future, with a neutrino factory the likely follow-on (some two decades hence) to LBNF. The work briefly summarized and cited here will have paved the way to these future machines. Smaller-scale implementations of cooled muon beams have also been discussed [61] and might proceed on other grounds.

Following nearly two decades of inspired work by the Muon Collaboration, the Neutrino Factory and Muon Collider Collaboration [62], ${ }^{3}$ Muons, Inc. [63], the Muon Collider Task Force [64], and now the Muon Accelerator Program, the P5 committee has recommended the termination of this effort albeit, with possible support for ongoing concept (though not technology) development through the DOE's General Accelerator R\&D program. The quest to do more with muons remains close to the hearts of its devotees, and physics soon to be discovered may yet have the last word.

\section{Acknowledgments}

It is a pleasure to acknowledge the many MC, NFMCC, Muons, Inc., MCTF, MICE, and MAP collaborators, too numerous to name here, without whom the work described could not have taken place, as well as the conference organizers who so kindly invited me to participate in ICNFP2014. This work is supported by the US Dept. of Energy (via the Muon Accelerator Program) and by the National Science Foundation under award PHY-1314008.

\section{References}

[1] R. B. Palmer, J. C. Gallardo, in Proc. XXVIII Int. Conf. on High Energy Physics, ed. Z. Ajduk, A. K. Wroblewski (World Scientific, Singapore, 1997), p. 435.

[2] T. Han, Z. Liu, Phys. Rev. D 87 (2013) 033007.

[3] V. Barger et al., "Particle physics opportunities at $\mu^{+} \mu^{-}$colliders," Nucl. Phys. B (Proc. Suppl.) 51A (1996) 13.

[4] V. Barger et al., "Physics of Higgs Factories," Proc. APS / DPF / DPB Summer Study on the Future of Particle Physics (Snowmass 2001), SNOWMASS-2001-E110.

[5] D. Neuffer, "The First Muon Collider - 125 GeV Higgs Factory?", AIP Conf. Proc. 1507, p. 849 (2012);

D. Cline, X. Ding, J. Lederman, “Higgs Boson Muon Collider Factory: $h^{0}, A, H$ Studies," Proc. IPAC'12, paper MOPPC042 (2012).

[6] ATLAS and CMS Collaborations, "Birth of a Higgs boson," CERN Cour., Apr. 26, 2013, http://cerncourier.com/cws/article/cern/53086.

[7] S. Geer, Phys. Rev. D 57, 6989 (1998); ibid. 59, 039903E (1999);

C. Albright et al., Fermilab-FN-692 (May 2000);

M. Apollonio et al., CERN-TH-2002-208 (Oct. 2002);

M. Lindner, in Neutrino Mass, ed. G. Altarelli, K. Winter, Springer Tracts in Modern Physics 190, 209 (2003).

\footnotetext{
${ }^{2} e^{+} e^{-}$colliders being less suited to such a mass range: CLIC, aimed at this mass range, has been shown to be immensely complex and expensive both to build and to operate, as well as suffering from beamstrahlung as discussed above.

${ }^{3}$ As the Muon Collaboration renamed itself upon realization of the power of the neutrino factory concept.
} 
[8] R. Abrams et al. (IDS-NF Collaboration), "International Design Study for the Neutrino Factory, Interim Design Report," arXiv:1112.2853 [hep-ex] (Mar. 2011).

[9] G. Gregroire et al., “An International Muon Ionization Cooling Experiment (MICE)," Proposal to Rutherford Appleton Laboratory, http://mice.iit.edu/micenotes/public/pdf/MICE0021/MICE0021.pdf; MICE website: http://mice.iit.edu.

[10] J.-P. Delahaye et al. (eds.), "Enabling Intensity and Energy Frontier Science with a Muon Accelerator Facility in the U.S.: A White Paper Submitted to the 2013 U.S. Community Summer Study of the Division of Particles and Fields of the American Physical Society," arXiv:1308.0494 [physics.acc-ph] (2013).

[11] See, e.g., M. A. Palmer, "An Overview of the US Muon Accelerator Program," Proc. COOL'13, Mürren, Switzerland, June 2013, paper MOAM2HA02 (2013);

MAP website: http://map. fnal .gov.

[12] P. Kyberd et al., "nuSTORM: Neutrinos from STORed Muons," arXiv:1206.0294 (2012).

[13] Particle Physics Project Priorization Panel, "Building for Discovery: Strategic Plan for U.S. Particle Physics in the Global Context,"

http://science.energy.gov/ /media/hep/hepap/pdf/May\%202014/ FINAL_P5_Report_053014.pdf.

[14] R. B. Palmer, "Muon Colliders," Rev. Accel. Sci. Tech. 7 (2014) 1 (to appear).

[15] K.T. McDonald et al., "Target System Concept for a Muon Collider/Neutrino Factory," Proc. IPAC2014, paper TUPRI008 (2014).

[16] K.T. McDonald et al., "The Merit High-Power Target Experiment at the CERN PS," Proc. IPAC'10, paper WEPE078 (2010).

[17] X. Ding, H.G. Kirk, K.T. McDonald, "Carbon Target Optimization for a Muon Collier/Neutrino Factory with a $6.75 \mathrm{GeV}$ Proton Driver,” Proc. IPAC2014, paper THPRI089 (2014);

R.J. Weggel et al., "Design of Magnets for the Target and Decay Region of a Muon Collider/Neutrino Factory Target," Proc. IPAC2013, paper TUPFI073 (2013).

[18] H. K. Sayed et al., "Optimizing Muon Capture and Transport for a Neutrino Factory/Muon Collider Front End," Proc. IPAC2013, paper TUPFI075 (2013).

[19] J.-P. Delahaye et al., "A Staged Muon Accelerator Facility for Neutrino and Collider Physics," Proc. IPAC2014, paper WEZA02 (2014).

[20] S. A. Bogacz, "Beam Dynamics of Low Energy Muon Acceleration," Nucl. Phys. B Proc. Supp. 155, 334 (2006).

[21] S. A Bogacz, "Maximizing Number of Passes in Muon RLA," AIP Conf. Proc. 981, 324 (2008).

[22] V. S. Morozov et al., "Linear Fixed-field Multipass Arcs for Recirculating Linear Accelerators," Phys. Rev. ST Accel. Beams 15, 060101 (2012).

[23] D. J. Summers et al., "Test of a 1.8 Tesla, $400 \mathrm{~Hz}$ Dipole for a Muon Synchrotron," Proc. IPAC2012, paper THPPD020 (2012).

[24] H. Witte et al., "Rapid Cycling Dipole Magnet," Proc. NA-PAC13, paper TUPRO115 (2013); H. Witte, J. S. Berg, M. L. Lopes, "Progress on the Dipole Magnet for a Rapid Cycling Synchrotron," Proc. IPAC2014, paper TUPRO115 (2014).

[25] D. J. Summers et al., "Muon Acceleration to $750 \mathrm{GeV}$ in the Tevatron Tunnel for a $1.5 \mathrm{TeV} \mu^{+} \mu^{-}$ Collider," Proc. PAC07, paper THPMS082 (2007).

[26] J. S. Berg, A. A. Garren, "Hybrid Fast-Ramping Accelerator to $750 \mathrm{GeV} / c$ : Refinement and Parameters over Full Energy Range," Technical Report BNL-98171-2012-IR, MAP-doc-4335 (2012). 
[27] D. Finley, N. Holtkamp (eds.), "Feasibility Study of a Neutrino Source Based on a Muon Storage Ring,” Report FERMILAB-PUB-00/108-E (2000).

[28] S. Ozaki, R. Palmer, M. Zisman, J. Gallardo eds., "Feasibility Study-II of a Muon-Based Neutrino Source," Report BNL-52623, June 2001, available from http://www . cap.bnl.gov/mumu/studyii/FS2-report.html

[29] A.V. Zlobin et al., "Storage Ring and Interaction Region Magnets for a $\mu^{+} \mu^{-}$Higgs Factory," Proc. PAC2013, paper THPBA19 (2013);

A.V. Zlobin et al., "Preliminary Design of a Higgs Factory $\mid m u^{+} \mu^{-}$Storage Ring," Proc. IPAC2013, paper TUPFI061 (2013).

[30] Y. Alexahin, E. Gianfelice-Wendt, A. Netepenko, "Conceptual design of the muon collider ring lattice," Proc. IPAC'10, paper TUPEB021 (2010).

[31] Y. Alexahin, E. Gianfelice-Wendt, “A 3-TeV muon collider lattice design," Proc. IPAC 2012, paper TUPPC041 (2012).

[32] Y. M. Ado, V. I. Balbekov, "Use of ionization friction in the storage of heavy particles," At. Energ. 31(1) 40 (1971), English translation in Atomic Energy (Springer) 31(1) 731, http: //www . springerlink. com/content/v766810126338571/.

[33] D. Neuffer, AIP Conf. Proc. 156, p. 201 (1987); D. Neuffer, " $\mu^{+} \mu^{-}$Colliders," Yellow Report CERN-99-12 (1999);

R. C. Fernow, J. C. Gallardo, Phys. Rev. E 52, 1039 (1995).

[34] A. A. Zholents, M. Zolotorev, W. Wan, Phys. Rev. ST Accel. Beams 4, 031001 (2001).

[35] S. Nagaitsev et al., "Design and Simulation of IOTA - a Novel Concept of Integrable Optics Test Accelerator," Proc. IPAC'12, paper MOYCP01 (2012).

[36] Y. Bao, A. Caldwell, D. Greenwald, C. Blume, "Frictional cooling demonstration at MPP," Proc. COOL2009 Workshop, paper TUM1MCCO03 (2009);

T. J. Roberts, D. M. Kaplan, “Particle Refrigerator,” Proc. PAC’09, paper WE6PFP096 (2009); M. Mühlbauer et al., Hyperfine Interact. 119, 305 (1999).

[37] Ya. Derbenev, R. P. Johnson, "Six-dimensional muon beam cooling using a homogeneous absorber: Concepts, beam dynamics, cooling decrements, and equilibrium emittances in a helical dipole channel," Phys. Rev. ST Accel. Beams 8, 041002 (2005).

[38] D. M. Kaplan, Proc. COOL'03 Workshop, Nucl. Instrum. Meth. A 532 (2004) 241.

[39] J. Beringer et al. (Particle Data Group), Phys. Rev. D 86, 010001 (2012).

[40] K. Yonehara et al., Proc. IPAC'13, paper TUPFI05 (2013);

D. Bowring et al., Proc. IPAC'12, paper THPPC033 (2012);

Y. Torun et al., Beam Dyn. Newslett. 55 (Aug. 2011) 103.

[41] MuCool Test Area website: http://mice.iit.edu/mta/.

[42] See e.g. D. V. Neuffer, C. Yoshikawa, "Muon Capture for the Front End of a $\mu^{+}-\mu^{-}$Collider," Proc. Pac2011, paper MOP030 (2011), and

J. S. Berg et al., "Cost-effective design for a neutrino factory," Phys. Rev. ST Accel Beams 9, 011001 (2006).

[43] Y. Alexahin, "Circularly Inclined Solenoid Channel for 6D Ionization Cooling of Muons," Proc. PAC09, paper TU3PBC04 (2009).

[44] C. Yoshikawa et al., "A Charge Separation Study to Enable the Design of a Complete Muon Cooling Channel," Proc. PAC2013, paper THPHO19 (2013).

[45] D. Stratakis, R. B. Palmer, D. P. Grote, "Space-charge Studies for Ionization Cooling Lattices," Proc. IPAC2013, paper TUPFI088 (2013). 
[46] D. Stratakis, R. C. Fernow, J. S. Berg, R. B. Palmer, “Tapered channel for six-dimensional muon cooling towards micron-scale emittances," Phys. Rev. ST Accel. Beams 16, 091001 (2013);

P. Snopok, G. G. Hanson, R. B. Palmer, "Simulations of the Tapered Guggenheim 6D Cooling Channel for the Muon Collider," Proc. PAC2011, paper MOP059 (2011);

P. Snopok, G. G. Hanson, "Six-Dimensional Cooling Lattice Studies for the Muon Collider," Proc. IPAC10, paper WEPE080 (2010);

P. Snopok, G. G. Hanson, “6D Cooling Simulations for the Muon Collider," Proc. PAC2009, paper FR5PFP035 (2009);

P. Snopok, G. G. Hanson, A. Klier, "Recent Progress on the 6D Cooling Simulations in the Guggenheim Channel,” Int. J. Mod. Phys. A 24, 987 (2009).

[47] R. Palmer et al., "Ionization cooling ring for muons," Phys. Rev. ST Accel. Beams 8, 061003 (2005).

[48] D. Stratakis, R. B. Palmer, J. S. Berg, H. Witte, "Complete 6-Dimensional Muon Cooling Channel for a Muon Collider,” Proc. IPAC2014, paper TUPME020 (2014).

[49] K. Yonehara, "Progress of HCC Design and Simulation," presented at MAP 2014 Spring Meeting, available at https://indico.fnal.gov/getFile.py/access?contribId=81 \&sessionId=2\&resId=1\&materialId=slides\&confId=8326 (2014).

[50] G. Flanagan et al., "Helical Muon Beam Cooling Channel Engineering Design," Proc. IPAC2013, paper THPBA22 (2013).

[51] P. Hanlet et al., "High Pressure RF Cavities in Magnetic Fields," Proc. EPAC 2006, p. 1364, June 2006.

[52] B. Freemire, "High Pressure Gas Filled RF Cavity Beam Test at the Fermilab MuCool Test Area," PhD Thesis, Illinois Institute of Technology (2013).

[53] H. K. Sayed, J. S. Berg, R. B. Palmer, D. Stratakis, "Design and Simulation of a High Field Low Energy Muon Ionization Cooling Channel,” Proc. IPAC14, paper TUPME019;

R. B. Palmer, R. C. Fernow, J. Lederman, "Muon Collider Final Cooling in 30-50 T Solenoids," Proc. PAC11, paper THOBN2 (2011).

[54] U. P. Trociewitz, "Another world record for the Magnet Lab: Research team generates worldrecord 35.4 tesla magnetic field using a superconducting insert magnet,"

http://www . magnet.fsu.edu/mediacenter/news/pressreleases/2011/

20110ct-27.html.

[55] Y. Shiroyanagi et al., "15+ T HTS Solenoid for Muon Accelerator Program," Proc. IPAC2012, paper THPPD048 (2012).

[56] J.A. Maloney et al., "Numerical Studies of Optimization and Aberration Correction Methods for the Preliminary Demonstration of the Parametric Ionization Cooling (PIC) Principle in the Twin Helix Muon Cooling Channel," arXiv:1401.8256 [physics.acc-ph] (2014) and references therein.

[57] D. Summers, private communication.

[58] M. Ellis et al., "The design, construction and performance of the MICE scintillating fibre trackers," Nucl. Instrum. Meth. A 659, 136 (2011).

[59] F. F. Tikhonin, “On the Effects with Muon Colliding Beams," JINR Report P2-4120 (Dubna, 1968);

G. I. Budker, "Accelerators and Colliding Beams," in Proc. 7th Int. Conf. on High-Energy Accelerators (Yerevan, 1969); extract available in AIP Conf. Proc. 352, 4 (1996).

[60] D. V. Neuffer, R. B. Palmer, "A high-energy high-luminosity $\mu^{+}-\mu^{-}$collider," Proc. 1994 Eur. Particle Accelerator Conf. (EPAC94), p. 52; 
J. C. Gallardo et al., "Muon Muon Collider: Feasibility Study," prepared for 1996 DPF/DPB Summer Study on New Directions in High-Energy Physics (Snowmass96), available from http://www . cap.bnl.gov/mumu/pubs/snowmass96.html;

C. M. Ankenbrandt et al., "Status of Muon Collider Research and Development and Future Plans,” Phys. Rev. ST Accel. Beams 2, 081001 (1999);

D. Ayres et al., "Expression of Interest in R\&D towards a Neutrino Factory Based on a Storage Ring and a Muon Collider," arXiv:physics/9911009;

M. M. Alsharo'a et al., "Recent Progress in Neutrino Factory and Muon Collider Research within the Muon Collaboration,” Phys. Rev. ST Accel. Beams 6, 081001 (2003).

[61] C. Y. Yoshikawa et al., "Intense Stopping Muon Beams," Proc. PAC'09, paper MO6RFP080 (2009).

[62] Neutrino Factory and Muon Collider Collaboration (NFMCC) website:

http://www. cap.bnl.gov/mumu/.

[63] Muons, Inc. website: http://muonsinc. com.

[64] Muon Collider Task Force (MCTF) website: https://mctf. fnal .gov/. 